\title{
Editorial: special issue on marine robotics
}

\author{
Giacomo Marani · Gianluca Antonelli • \\ Hyun-Taek Choi
}

Published online: 15 September 2011

(c) Springer-Verlag 2011

The marine environment is an exciting and challenging context for the robotics community. Autonomous underwater and surface vehicles face a demanding environment with poor perception and actuation capabilities with respect to the ground-based robotics. In case of the underwater scenario, for example, communications are complicated by the limited bandwidth, and the position measurement of the vehicles is not obvious. Moreover, the presence of current and wind disturbances enhances the complexity of the dynamic control tasks.

With the above difficulties, a successful mission must be supported by the most advanced robot technologies, with particular attention to autonomy. The marked progress of the last decade in marine technologies and robotics has led to massive improvements of marine robotics. The state of the art and some achievements in this area are particularly innovative.

The ocean covers about two-thirds of the earth and has a great effect on the future existence of all human beings. In the near future, marine robots will be used for homeland security, for rise inspection or intelligent sampling, and researchers will be able to deploy numerous networked robotic ocean observatories, expanding the quantity and quality of data

\footnotetext{
G. Marani $(\bowtie)$

WVU-NASA Robotics Center, 1000 Galliher Drive, Fairmont, WV 26554, USA

e-mail: Giacomo.Marani@mail.wvu.edu

G. Antonelli

Università di Cassino, via G. Di Biasio 43,

03043 Cassino (FR), Italy

e-mail: antonelli@unicas.it

H.-T. Choi

Maritime and Ocean Engineering Research Institute, KORDI, Daejeon 305-343, Republic of Korea

e-mail: htchoi@moeri.re.kr
}

available to scientists and the public worldwide. The introduction of advanced technologies, like autonomous manipulation will enable sensing and performing mechanical work in hazardous areas or those inaccessible to humans, such as natural or man-made disastrous regions, deep ocean, and under ice.

Given some similarities of problems encountered in autonomous underwater robotic tasks and space intervention missions, marine robotics is also regarded as a valid reference for developing strategies in future space robotic missions.

The primary goal of this special issue is to present recent advances in unmanned underwater (UUVs) and surface (USVs) robotic vehicle technologies. This special issue is organized by the Marine Robotics Technical Committee of the IEEE Robotics and Automation Society, and co-edited by Gianluca Antonelli (University of Cassino, Italy), Hyun T. Choi (Maritime \& Ocean Engineering Research Institute, Korea) and Giacomo Marani (WVU-NASA Robotics Center, USA). This issue, thorough the presentation of five peerreviewed papers, covers several topics of the area.

Junku Yuh et al., with their introductory paper, present some exemplary models of recent developments in marine robots in different application areas. Jenna Brown et al. introduce the use of autonomous vehicles (AVs) in estuarine and riverine environments. Small, versatile, efficient, moving platforms like AVs have been equipped with a suite of instruments for measuring environmental conditions.

One potential application of AUVs is for the purpose of undersea surveillance. Stephanie Kemna et al., in their paper, present a recent AUV implementation for active antisubmarine warfare tested at sea in the summer of 2010.

Distributed acoustic localization of teams of autonomous underwater vehicles is the topic of Giuseppe Casalino et al. They proposes a solution for evaluating the distance between any pair of AUVs in the team. 
Finally, Laszlo Techy presents recent advances in timeoptimal navigation for flight vehicles in a planar, timevarying flow field, where the objective is to find the fastest trajectory between the initial and final points.

The guest editors would like to thank the authors, reviewers and the editorial team for their contribution and services to this special issue. I would also like to thank the former editor in chief of the Intelligent Service Robotics journal,
Dr. Junku Yuh, for his fundamental role in the development of this publication and the new EIC, Prof. Il Hong Suh, for his support and advices in the review and editorial process.

Given the vastness of the topics related to marine robotics, an exhaustive presentation would certainly require more than a single issue, but we hope that this introduction of the most recent research will stimulate further interests and discussions in this fascinating field. 